\title{
Determination of the fatty-acid composition of four native microalgae species
}

\author{
Şahin Oya Irmak ${ }^{1, *}$ and Akpınar-Bayizit Arzu ${ }^{2}$ \\ ${ }^{1}$ Chemical Engineering Department, Faculty of Engineering, Yalova University, Yalova, Turkey. \\ ${ }^{2}$ Food Engineering Department, Faculty of Agriculture, Uludag University, Bursa, Turkey.
}

Publication history: Received on 23 June 2020; revised on 07 July 2020; accepted on 08 July 2020

Article DOI: https://doi.org/10.30574/gscarr.2020.4.1.0053

\begin{abstract}
In this study, two green (Chlorella sp. and Ankistrodesmus sp.) and two blue-green (Oscillatoria sp. and Chroococcus sp.) species of microalgae, which were obtained from a native microalgae culture collection from Turkey, were cultivated and investigated via capillary GC to determine their fatty-acid profiles. Growth media were chosen with consideration of the biological properties of each algae species. Our results, as well as other published data, show that fatty-acid composition offers discriminating features that allow the chemotaxonomic classification of these algae. Fatty-acid profiles and lipid contents in microalgal biomass varied between species. The microalgae containing the highest proportion of lipid in its biomass was in Chroococcus sp. (1.72\%), while that containing the lowest was Ankistrodesmus sp. (0.14\%). The major fatty acids in Chlorella sp. were C18:0 (26.70\%), C18:1 (23.48 $\pm 0.0005 \%)$ and C16:0 (21.05\%). C18:1 was the major fatty acid in the other three species of microalgae: Ankistrodesmus sp. (36.83 $\pm 0.0007 \%)$, Oscillatoria sp. (38.51\%) and Chroococcus sp. (47.96\%). EPA, a nutritionally important polyunsaturated fatty acid, was not observed in Chlorella sp. but was detected in Ankistrodesmus sp., Oscillatoria sp. and Chroococcus sp. at proportions of $0.35 \%, 0.30 \%$ and $0.62 \%$, respectively. In addition, another essential PUFA, DHA, was observed in all microalgae species in varying amounts.
\end{abstract}

Keywords: Ankistrodesmus; Chlorella; Chroococcus; Oscillatoria; Fatty acid; Microalgae

\section{Introduction}

Algae, a group of autotrophic and eukaryotic organisms, have been used for centuries as a food by native peoples, especially in Asian countries, such as China, Japan and Korea. This has led to the utilization of algae as a food supplement for undernourished people in many parts of the world, due to their high protein content ( $25 \%$ ), high digestibility and specific amino-acid content. Algae can produce organic compounds, such as polyunsaturated fatty acids, amino acids, vitamins, etc., with the aid of simple inorganic sources when growth parameters, e.g., nutrient quantity and quality, light, $\mathrm{pH}$, turbulence, salinity and temperature, are optimized.

Polyunsaturated fatty acids (PUFAs) are fatty acids of 18 carbons or more in length containing two or more methyleneinterrupted double bonds in the cis position, and they are essential nutrients for humans that can only be obtained from food. These fatty acids can be grouped into two main families, $\omega-6$ (or n-6) and $\omega-3$ (or n-3), depending on the position of the first double bond nearest to the methyl end of the fatty acid. Both $\omega-6$ and $\omega$ - 3 long-chain polyunsaturated fatty acids (LC-PUFAs) are structural components of cell membranes that modulate membrane fluidity and permeability $[1,2]$.

Additionally, LC-PUFAs serve as precursors for several biologically active molecules, such as eicosanoids, growth regulators and hormones, which exhibit hormonal and immunological activity [3].

\footnotetext{
* Corresponding author: Şahin Oya Irmak
} 
The properties of LC-PUFAs have recently garnered considerable interest because they have numerous nutraceuticals and pharmaceutical applications [4,5]; in particular, the vascular- protective effects of long-chain $\omega-3$ fatty acids are well-documented. Eicosapentaenoic acid (EPA, C20:5 $\omega$-3) and docosahexaenoic acid (DHA, C22:6 $\omega-3$ ) are known to affect the human lipid profile, vascular tone and blood coagulation and have clinical importance in the treatment of atherosclerosis, cancer, rheumatoid arthritis, psoriasis and diseases such as Alzheimer's [6]. Arachidonic acid (ARA, $\mathrm{C} 20: 4 \omega-6)$ and DHA are especially vital in the brain and blood vessels, are involved in the development and maturation of neuronal structures, and have been shown to be essential throughout the entire life span for the maintenance of normal nervous system function, as well as post-natal brain and retina development [7-9].

The $\omega-6$ and $\omega-3$ PUFAs are derived from linoleic acid (LA, C18:2 $\omega-6)$ and $\alpha$-linolenic acid (ALA, C18:3 $\omega-3$ ), respectively. Humans are incapable of synthesizing these two fatty acids due to a lack of the $\Delta 12$ - and $\Delta 15$-desaturases. However, humans can metabolize these two fatty acids once they are obtained from the diet and subsequently form longer and more unsaturated PUFAs through a series of desaturation and elongation steps.

The principal dietary sources of PUFAs are marine fish oils and plant seed oils. Because recovering PUFAs from fish oil is expensive and plant sources are limited, alternative sources are being studied extensively [10]. Microbial lipids with compositions similar to plant oils and animal fats have often been considered as substitute oil and fat resources [11]. Microorganisms are considered to be abundant sources of oil and fats because their membranes and membranous structures always contain lipids [12].

Microalgae are known to synthesize multi-bioactive compounds, including fatty acids, proteins and amino acids, among others. Microalgae produce high levels of $\omega-3$ PUFAs and are promising sources for production of selected fatty acids with large-scale culture technologies [13]. Algal fatty acids can be obtained through photoautotrophic, heterotrophic and mixotrophic cultivation techniques. Although photoautotrophic production is often limited by insufficient light, a result of the shade caused by cell growth, algal oil has been successfully produced based on photoautotrophic growth $[14,15]$. Much effort is being devoted to developing a commercially feasible technology to produce "oil" directly from microalgae [15]. As the prices for most bulk plant oils are relatively low, and animal fats are even cheaper, it is unlikely that microbial production processes for oils resembling these 'common' oils can ever be commercially viable. Evidently, to develop economically feasible oil production processes for cultivation of oleaginous microalgae in bioreactors, it is important that the oils or fatty acids are produced at a high rate and are of sufficiently high value, i.e., they contain a high proportion of PUFAs or a specific PUFA. In this paper, fatty-acid profiles of four native green and blue-green microalgae were reported.

\section{Material and methods}

\subsection{Materials}

Algal cultures were supplied from GAZI-MACC (Collection of Tahir Atici, Gazi University Microalgae Culture Collection, Ankara, Turkey). The green algal strains examined were Chlorella sp. and Ankistrodesmus sp., and the blue-green algal strains were Oscillatoria sp. and Chroococcus sp. (Figure 1).

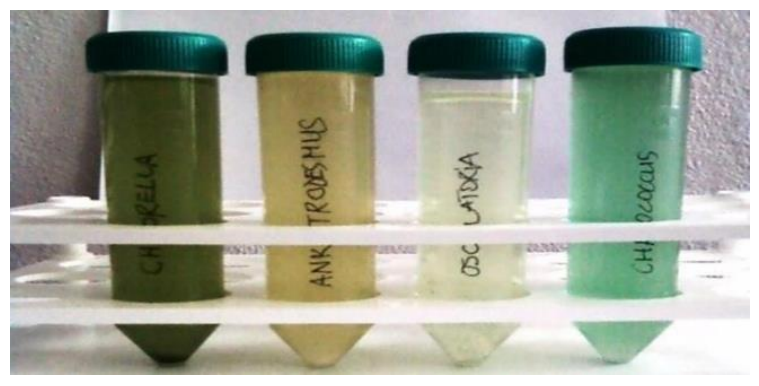

Figure 1 The examined native microalgae species' stock cultures

The cells were pre-cultivated in $250 \mathrm{~mL}$ Erlenmeyer flasks containing $50 \mathrm{~mL} \mathrm{BG-11} \mathrm{Medium} \mathrm{(for} \mathrm{the} \mathrm{blue-green} \mathrm{algae)}$ or Bold's Basal Medium (for the green algae) for a period of 15 days. After pre-cultivation, cells were transferred into 2 L Erlenmeyer flasks containing 1000 mL BG-11 or Bold's Basal, which were shaken orbitally at $150 \mathrm{rpm}$ in a shaker incubator. The incubation was carried out at $28 \pm 2{ }^{\circ} \mathrm{C}$ for 30 days with continuous illumination from above at a light rate of $12: 12(\mathrm{~h} / \mathrm{h})$. Throughout the incubation, the growth period and algal cell optical density of each microalgae 
species were obtained by spectrophotometric measurement, as described by Liang et al [16], based on the cell density during cultivation.

\subsection{Harvest and analysis}

Algal cells were harvested in the stationary phase by vacuum filtration through a double thickness of Whatman GF/A filter paper, then washed with distilled water $(2 \times 500 \mathrm{~mL})$. The method of Folch et al. [17] was used for lipid extraction. Approximately $5 \mathrm{~g}$ wet cells were extracted into $150 \mathrm{~mL}$ chloroform/methanol mixture $(2: 1, \mathrm{v} / \mathrm{v})$ at room temperature for $24 \mathrm{~h}$. Cell debris was removed from the organic phase by filtration and washed with $50 \mathrm{~mL}$ chloroform/methanol $(2: 1, v / v)$. The two organic extracts were transferred into a separating funnel and washed twice with $100 \mathrm{~mL}$ of distilled water. After each washing, the organic phase was retained. Residual water was removed with anhydrous magnesium sulphate (MgSO4) and the solvent was evaporated under vacuum until absolute dryness was achieved. Lipid material was dissolved in a minimal volume of diethyl ether and quantitatively transferred to a pre-weighed vial. The ether was subsequently removed under a stream of nitrogen. The sample was dried in a vacuum desiccator over phosphorus pentoxide $\left(\mathrm{P}_{2} \mathrm{O}_{5}\right)$ for $24 \mathrm{~h}$, then reweighed.

Cold esterification was carried out to obtain fatty-acid methyl esters (FAMEs) of the algal lipids according to the International Union of Pure and Applied Chemistry (IUPAC) method. The FAMEs were analysed using a gas chromatograph (Agilent 6890N, Hewlett-Packard Co., USA) equipped with a flame ionization detector and a capillary column (Agilent DB23 column) operating on a split mode. The oven temperature was programmed as follows: the initial temperature, $130{ }^{\circ} \mathrm{C}$, was raised to $170{ }^{\circ} \mathrm{C}$ at a rate of $6.5^{\circ} \mathrm{C} / \mathrm{min}$ and was held at this temperature for $1 \mathrm{~min}$; then, it was increased at a rate of $2.15^{\circ} \mathrm{C} / \mathrm{min}$ to $215^{\circ} \mathrm{C}$, held at this temperature for $12 \mathrm{~min}$, and then increased to $230{ }^{\circ} \mathrm{C}$ and held at this temperature for $3 \mathrm{~min}$. Nitrogen was used as the carrier gas, and the sample injection volume was $1 \mu \mathrm{L}$. Peaks were identified by comparing their retention times to authentic standards analysed under the same conditions. Peak areas resulting from triplicate injections were measured with an HP computing integrator. Results were expressed as percent total fatty acids.

\section{Results and discussion}

The cell growth profiles of microalgae species were presented in Fig 2. Also, biomass weights and total lipid content were shown in Table 1.

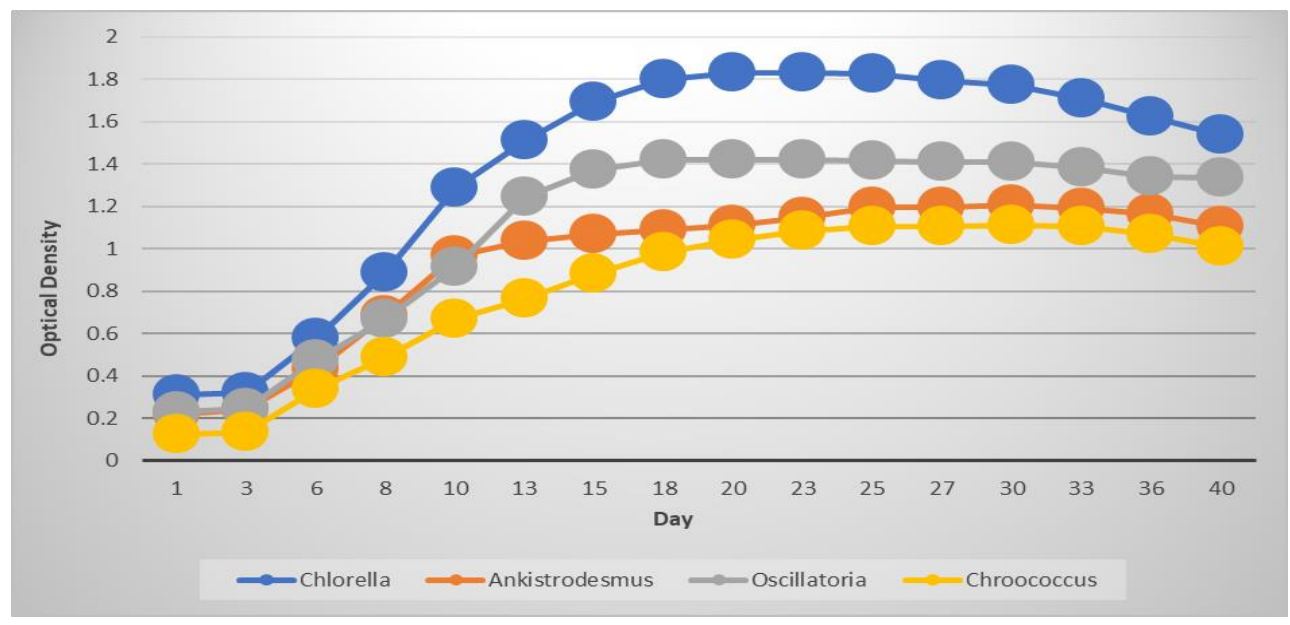

Figure 2 Growth profile of native microalgae species (optical density per day).

The fatty-acid composition of the total cellular lipids provides information that is potentially interesting for algal taxonomy. However, caution should be taken when interpreting the available information regarding fatty acid composition from algae. Many factors affect fatty-acid composition, such as growth stage, irradiance, temperature, nutrients, salinity and the depth under water during growth.

Dried algal samples always exhibit a lower PUFA content because these compounds are very susceptible to oxidation. The degree of PUFA loss depends on the species; texture; time of exposure to sunlight, high temperature or air; and storage time and conditions [18]. The results obtained by extracting fatty acids from freeze-dried samples that were kept in a dark, dry and cold place for months, as well as those from fresh samples stored in a freezer at a temperature 
less than $-70{ }^{\circ} \mathrm{C}$ over a period of months, still proved acceptable, but were not as good as the results obtained from freshly extracted algae. The true fatty acid profile can only be obtained from freshly collected algal samples that are then immediately extracted and analysed [19].

The biomass of Chlorella sp. was two-fold higher than that of Ankistrodesmus sp.; however, there was a three-fold difference in the total lipid contents of these two algae. Oscillatoria sp. was determined to have two-fold higher biomass than Chroococcus sp; however, conversely, the lipid content of Chroococcus sp. was two-fold higher. Lipid contents measured in the dry biomass of the examined microalgae species were between $1.24 \%$ and $15.13 \%$ (Table 1). Chroococcus sp. had the highest lipid content in its biomass, 15.13\%, which was similar to data previously reported for Chlorella sp. and Chroococcus sp. [20-22].

Table 1 Total lipid contents and biomass weights of microalgae species

\begin{tabular}{l|lll}
\hline \multicolumn{1}{l}{ Microalgae } & $\begin{array}{l}\text { Wet biomass } \\
\text { (g.l-1) }\end{array}$ & $\begin{array}{l}\text { Dry biomass } \\
\text { (g.l-1) }\end{array}$ & $\begin{array}{l}\text { Lipid* } \\
\text { (\%) }\end{array}$ \\
\hline Chlorella sp. & 21.9627 & 2.4958 & 3.58 \\
Ankistrodesmus sp. & 11.2871 & 1.2826 & 1.24 \\
Oscillatoria sp. & 16.2162 & 1.8428 & 8.36 \\
Chroococcus sp. & 8.3274 & 0.9463 & 15.13 \\
\hline \multicolumn{2}{c}{$*$ dry weight biomass }
\end{tabular}

Cells sampled during the stationary phase were used for fatty-acid analysis. The fatty-acid compositions of the total lipid contents, determined by capillary gas chromatography, as well as the total lipid contents of the four microalgae, are presented in Table 1. The results of the present investigation revealed differences in fatty-acid compositions among the four species of algae examined. The data indicated the presence of 26 fatty-acid compounds with different chain lengths, ranging from 8 to 24 carbons. Although polyunsaturated fatty acids were not the dominant fatty acids, their contents ranged from $3.36 \%$ in Chroococcus sp. to $17.61 \%$ in Chlorella sp. The content of unsaturated fatty acids was higher in the blue-green algae species than in the green algae species.

Table 2 Saturated fatty acid profiles of native microalgae species

\begin{tabular}{l|llll}
\hline (\%) & Chlorella sp. & Ankistrodesmus sp. & Oscillatoria sp. & Chroococcus sp. \\
\hline C8:0 & nd & $1.01 \pm 0.0014$ & nd & $0.39 \pm 0.0004$ \\
C10:0 & nd & $0.24 \pm 0.0002$ & nd & nd \\
C12:0 & $0.24 \pm 0.0006$ & $0.25 \pm 0.0004$ & $0.26 \pm 0.0007$ & $0.37 \pm 0.0004$ \\
C14:0 & $0.94 \pm 0.0001$ & $0.99 \pm 0.0005$ & $0.89 \pm 0.0004$ & $1.24 \pm 0.0005$ \\
C15:0 & $0.44 \pm 0.0007$ & $0.45 \pm 0.0014$ & $0.43 \pm 0.0002$ & $0.85 \pm 0.0007$ \\
C16:0 & $21.05 \pm 0.0089$ & $19.94 \pm 0.0011$ & $15.87 \pm 0.0011$ & $6.99 \pm 0.0009$ \\
C17:0 & $1.40 \pm 0.0042$ & $0.44 \pm 0.0056$ & $0.43 \pm 0.0005$ & nd \\
C18:0 & $26.70 \pm 0.0001$ & $19.83 \pm 0.0028$ & $15.83 \pm 0.0011$ & $12.85 \pm 0.0011$ \\
C20:0 & $0.65 \pm 0.0002$ & trace & trace & $1.28 \pm 0.0014$ \\
C22:0 & $0.28 \pm 0.0001$ & $1.34 \pm 0.0004$ & nd & $0.65 \pm 0.0001$ \\
C23:0 & $0.68 \pm 0.0002$ & $0.35 \pm 0.0004$ & $1.03 \pm 0.0014$ & $1.10 \pm 0.0002$ \\
C24:0 & trace & $0.30 \pm 0.0007$ & trace & nd \\
SFA & 52.38 & 45.14 & 34.74 & 25.72 \\
\hline
\end{tabular}


In the analysed microalgae species, stearic acid (C18:0) and palmitic acid (C16:0) were the most abundant saturated fatty acids (Table 2). The total proportions of saturated fatty acids (SFAs) and monounsaturated fatty acids represented $25.72 \%$ to $52.38 \%$ and $28.87 \%$ to $70.92 \%$ of the total fatty acids, respectively. The proportion of all SFAs was considerably higher in the green algae species (Chlorella sp. and Ankistrodesmus sp.) than in the blue-green species. The content of C18:0 was highest in Chlorella sp., representing 26.70\% of the total FAMEs, and lowest in Chroococcus sp., representing $12.85 \%$ of the total FAMEs. Palmitic acid content varied from $6.99 \%$ to $21.05 \%$. Lauric acid (C12:0), myristic acid (C14:0), pentadecanoic acid (C15:0) and tricosacoic acid (C23:0) were detected in the algae species tested, but in small quantities. Caprylic acid (C8:0), capric acid (C10:0), heptadecanoic acid (C17:0), eicosanoic acid (C20:0), docosanoic acid (C22:0) and tetracosanoic acid (C24:0) were found either in trace levels or not detected. It is interesting to note that the average percentage of these minimally detected saturated fatty acids was slightly higher in Chroococcus sp. compared to Oscillatoria sp. or either of the green algae species.

Among the monounsaturated fatty acids (MUFAs), oleic acid (C18:1, cis-9) was the most abundant fatty acid in all algae examined; the observed proportions of oleic acid ranged from $23.48 \%$ to $47.96 \%$, with an average of $36.70 \%$ (Table 3 ).

High concentrations of oleic acid were observed in the blue-green algae and Ankistrodesmus sp., representing more than $35 \%$ of the total FAMEs of these species. The total content of MUFAs was $60.63 \%$ to $70.92 \%$ in the blue-green algae and was found to be higher in Ankistrodesmus sp. than in Chlorella sp. Despite its relatively low biomass, Chroococcus sp. was found to produce the highest proportion of unsaturated fatty acids, $74.28 \%$, mainly consisting of monounsaturated (70.92\%). Oscillatoria sp., which exhibited a two-fold higher biomass production, was also a good source of MUFAs (60.63\%) [23]. Rezanka et al [24] and Patil et al [21] reported that palmitic acid was the dominant fatty acid in most algae, with proportions of $24.13 \%$ and $21.30 \%$ respectively. However, the results of this research contradicted that finding.

Table 3 Monounsaturated fatty acid profiles of native microalgae species

\begin{tabular}{l|llll}
\hline (\%) & Chlorella sp. & Ankistrodesmus sp. & Oscillatoria sp. & Chroococcus sp. \\
\hline C14:1, cis-9 & $0.50 \pm 0.0004$ & $1.25 \pm 0.0004$ & $3.63 \pm 0.0056$ & $0.91 \pm 0.0002$ \\
15:1, cis-10 & $0.37 \pm 0.0056$ & $0.48 \pm 0.0028$ & $8.41 \pm 0.0005$ & $8.54 \pm 0.0002$ \\
C16:1, cis-9 & $0.82 \pm 0.0014$ & $6.19 \pm 0.0042$ & $6.08 \pm 0.0014$ & $3.67 \pm 0.0014$ \\
C17:1, cis-7 & $1.26 \pm 0.0014$ & $0.37 \pm 0.0002$ & $0.57 \pm 0.0007$ & $1.12 \pm 0.0007$ \\
C18:1, cis-9 & $23.48 \pm 0.0005$ & $36.83 \pm 0.0007$ & $38.51 \pm 0.0056$ & $47.96 \pm 0.0039$ \\
C18:1, trans-9 & $2.19 \pm 0.0001$ & $1.66 \pm 0.0002$ & $2.09 \pm 0.0056$ & $7.81 \pm 0.0084$ \\
C20:1, cis-11 & $0.42 \pm 0.00003$ & $0.26 \pm 0.0004$ & $0.34 \pm 0.0002$ & $0.91 \pm 0.0005$ \\
MUFA & 28.87 & 47.31 & 60.63 & 70.92 \\
\hline
\end{tabular}

LA, ALA, eicosatrienoic acid (C20:3 all cis-5,8,11; $\omega$-9), EPA and DHA were the predominant PUFAs observed as seen in Table 4 . The quantity of LA averaged from $0.30 \%$ of the total FAMEs in Oscillatoria sp. to $1.46 \%$ of the total FAMEs in Chlorella sp. Algal fatty acid studies are based principally on EPA, DHA and ARA production [19,21,22,25-30].

Nitzschia sp., Crypthecodinium sp., Porphyridium sp., Schizochytium sp. and Chlorella sp. are the most widely studied species. In the present study, the corresponding PUFAs, i.e., the total content of polyunsaturated fatty acids with C18, C20 and C22, accounted for $3.36 \%$ to $17.61 \%$ of the total FAMEs, with the highest proportion observed in Chlorella sp. Contrary to the marine species of Chroococcus sp., which Patil et al. [21] determined an 11.9\%, the lowest PUFA proportion observed in the native fresh Chroococcus sp. There were large variations in the PUFA contents between all species examined. Eicosatrienoic acid and ARA were detected only in Chlorella sp. in very trace amounts. DHA was detected in all species in proportions from $0.28 \%$ to $2.22 \%$. The highest quantity of DHA was detected in Oscillatoria sp.

Ahlgren et al [31] reported that blue-green algae can synthesize $\omega$-3 fatty acids in higher quantities than green algae can. However, in the present study, among the four microalgae examined, the green algae species (Chlorella sp. and Ankistrodesmus sp.) were found to accumulate more $\omega-3$ fatty acids than the blue- green algae species, mainly due to comparatively greater synthesis of $\alpha$-linolenic acid (C18:2 all cis-9,12,15). 
Table 4 Polyunsaturated fatty acid profiles of microalgae species

\begin{tabular}{l|llll}
\hline (\%) & Chlorella sp. & Ankistrodesmus sp. & Oscillatoria sp. & Chroococcus sp. \\
\hline C18:2, CIS-9,12 & $1.46 \pm 0.0002$ & $0.39 \pm 0.0012$ & $0.30 \pm 0.0007$ & $0.35 \pm 0.0011$ \\
C18:3, CIS- 9,12,15 & $11.78 \pm 0.0004$ & $6.45 \pm 0.0070$ & $1.48 \pm 0.0014$ & $0.91 \pm 0.0005$ \\
C20:3, CIS- $5,8,11$ & $2.83 \pm 0.0002$ & nd & nd & ND \\
C20:4, CIS- $5,8,11,14$ & Trace & nd & nd & ND \\
C20:5, CIS- $5,8,11,14,17$ & Trace & $0.35 \pm 0.0007$ & $0.30 \pm 0.0005$ & $0.62 \pm 0.0002$ \\
C22:6, CIS- 4,7,10,13,16,19 & $1.54 \pm 0.0007$ & $0.28 \pm 0.0002$ & $2.22 \pm 0.0056$ & $1.48 \pm 0.0007$ \\
PUFA & 17.61 & 7.47 & 4.30 & 3.36 \\
\hline
\end{tabular}

A low ratio of $\omega-6$ to $\omega-3$ PUFAs has often been cited as an index of high nutritional value [32]. The ratios calculated from tables indicated the nutritional values to be 1.46: 13.32 for Chlorella sp, 0.39: 7.08 for Ankistrodesmus sp, 0.30: 4.00 for Oscillatoria sp, and 0.30: 3.01 for Chroococcus sp. The results of this study showed that the percentage of $\omega-6$ fatty acids synthesized by these algae is comparably low. Because most foods consumed in Europe possess a $\omega-6$ : $\omega-3$ ratio between $15: 1$ and 17:1, these values suggest that algae consumption could be beneficial for health. Furthermore, the occurrence of ALA and DHA in these algae is of considerable dietary value because the addition of these $\omega-3$ PUFAs to the diet has been reported to promote growth, strengthen cell membrane integrity, and improve heart function, circulatory function and mental health [33-35].

The proportion of $\omega-9$ fatty acids varied between $28.43 \%$ of the total FAMEs in Chlorella sp. and $61.26 \%$ of the total FAMEs in Chroococcus sp. Furthermore, the examined algae species contained $\omega-7$ fatty acids, as well as trans fatty acids. The analysis of the data revealed that in the green and blue-green algae species tested, approximately $98 \%$ of their total FAME contents consisted of long-chain fatty acids (LCFAs; longer than 12 carbons). In contrast, short-chain fatty acids (SCFAs; fewer than 6 carbons) were not detected in the algae species investigated. The proportions of medium-chain fatty acids (MCFAs; 8 to 12 carbons) were very low, varying between $0.39 \%$ to $1.25 \%$ of the total FAMEs, and no MCFAs were detected in Chlorella sp. and Oscillatoria sp. It is known that algae represent an important source of LCFAs that are fundamental for the formation of important structural lipids and elements of cell membranes.

\section{Conclusion}

Microalgae have long been considered as a promising feedstock for biomass production. On the other hand, they have been explored for their unique potential to yield a variety of biofuels concomitantly with generation of value-added products and phycoremediation of wastewater [36]. The cultivation of microalgae is a significant factor. The choice of cultivation system and conditions has to be emphasized because the phycoremediation efficiency and the yield of biofuels and other value-added products would largely depend on it. Also significant is the composition of fatty acids of the different microalgae species, as they can have a significant effect on the characteristics of biomass to be used.

The presented study was investigated the total lipid content and fatty-acid distribution of two green and two blue-green fresh microalgae species, which were isolated Turkey. Oleic acid was the most abundant fatty acid in all algae examined, followed by palmitoleic acid. The concentration of oleic acid was high in blue-green algae and Ankistrodesmus sp. Hexadecenoic acid and stearic acid were the major monounsaturated fatty acids observed. The fatty-acid contents varied remarkably among microalgae grown under the same heterotrophic culture processes. LA, ALA, EPA and DHA represented the predominant proportions of PUFAs. The low ratio of $\omega-6$ to $\omega-3$ PUFAs was noteworthy because it represents a high index of nutritional value. The investigation of these native microalgae verified the presence of several health-promoting and beneficial fatty acids, such as long-chain unsaturated fatty acids. However, consequence of fatty acid profiles showed that Chlorella sp. and Ankistrodesmus sp. biomasses were ideal for the biofuel potential, where Chroococcus sp. and Oscillatoria sp. were ideal for food and feed applications. 


\section{Compliance with ethical standards}

\section{Acknowledgments}

No funding has been received to support this work. No funds have been received, to cover the costs to publish in open access.

\section{Disclosure of conflict of interest}

The authors declare no conflict of interest.

\section{References}

[1] Bordoni A, Di Nunzio M, Danesi F and Biagi PL. (2006). Polyunsaturated fatty acids: From diet to binding to ppars and other nuclear receptors. Genes \& Nutrition, 1, 95-106.

[2] Schuchardt JP, Huss M, Stauss-Grabo M and Hahn A. (2010). Significance of long-chain polyunsaturated fatty acids (PUFAs) for the development and behaviour of children. European Journal of Pediatrics, 169, 149-164.

[3] Jump DB. (2002).The Biochemistry of n- 3 Polyunsaturated Fatty Acids Journal of Biological Chemistry, 277, 8755-8758.

[4] Shahidi F and Wanasundara UN. (1998). Omega-3 fatty acid concentrates: nutritional aspects and production technologies. Trends in Food Science \& Technology, 9, 230-240.

[5] Horrocks LA and Yeo YK. (1999). HEALTH BENEFITS OF DOCOSAHEXAENOIC ACID (DHA). Pharmacological Research, 40, 211-225.

[6] Simopoulos AP. (1999). Essential fatty acids in health and chronic disease. The American Journal of Clinical Nutrition, 70, 560-569.

[7] Erdman J, Oria M and Pillsbury L (Institute of Medicine (US) Committee on Nutrition). (2011). Eicosapentaenoic Acid (EPA) and Docosahexaenoic Acid (DHA). In: Erdman J, Oria M and Pillsbury L (Eds), Nutrition and Traumatic Brain Injury: Improving Acute and Subacute Health Outcomes in Military Personnel. The National Academic Press, Washington, DC, 188-204.

[8] Crawford MA. (2000). Placental delivery of arachidonic and docosahexaenoic acids: implications for the lipid nutrition of preterm infants. The American Journal of Clinical Nutrition, 71, 275-284.

[9] Uauy R and Dangour AD. (2008). Nutrition in Brain Development and Aging: Role of Essential Fatty Acids. Nutrition Reviews, 64, 24-33.

[10] Belarbi EH, Molina E and Chisti Y. (2000). A process for high yield and scaleable recovery of high purity eicosapentaenoic acid esters from microalgae and fish oil. Enzyme and Microbial Technology, 26, 516-529.

[11] Ratledge C and Wynn JP. (2002). The biochemistry and molecular biology of lipid accumulation in oleaginous microorganisms. Advances in Applied Microbiology, 51, 1-51.

[12] Sahena F, Zaidul ISM, Jinap S, Saari N, Jahurul HA, Abbas KA and Norulaini NA. (2009). PUFAs in Fish: Extraction, Fractionation, Importance in Health. Comprehensive Reviews in Food Science and Food Safety, 8, 59-74.

[13] Huerlimann R, Nys R de and Heimann K. (2010) Growth, lipid content, productivity and fatty acid composition of tropical microalgae for scale-up production. Biotechnology and Bioengineering, 107, 245-257.

[14] Sánchez Mirón A, Cerón García MC, García Camacho F, Molina Grima E and Chisti Y. (2002). Growth and biochemical characterization of microalgal biomass produced in bubble column and airlift photobioreactors: studies in fed-batch culture. Enzyme and Microbial Technology, 31, 1015-1023.

[15] Molina Grima E, Belarbi EH, Acién Fernández FG, Robles Medina A and Chisti Y. (2003). Recovery of microalgal biomass and metabolites: process options and economics. Biotechnology Advances, 20, 491-515.

[16] Liang Y, Sarkany N and Cui Y. (2009). Biomass and lipid productivities of Chlorella vulgaris under autotrophic, heterotrophic and mixotrophic growth conditions. Biotechnology Letters, 31, 1043-1049.

[17] Folch J, Lees M and Stanley GHS. (1957). A Simple Method for the Isolation and Purification of Total Lipides from Animal Tissues. Journal of Biological Chemistry, 226, 497-509. 
[18] Kołakowska A, Domiszewski Z, Kozłowski D and Gajowniczek M. (2006). Effects of rainbow trout freshness on n3 polyunsaturated fatty acids in fish offal. European Journal of Lipid Science and Technology, 108, 723-729.

[19] Jiménez Callejón MJ, Robles Medina A, González Moreno PA, Esteban Cerdán L, Orta Guillén S and Molina Grima E. (2020). Simultaneous extraction and fractionation of lipids from the microalga Nannochloropsis sp. for the production of EPA-rich polar lipid concentrates. Journal of Applied Phycology, 32, 1117-1128.

[20] Petkov G and Garcia G. (2007). Which are fatty acids of the green alga Chlorella? Biochemical Systematics and Ecology, 35, 281-285.

[21] Patil V, Källqvist T, Olsen E, Vogt G and Gislerød HR. (2007). Fatty acid composition of 12 microalgae for possible use in aquaculture feed. Aquaculture International, 15, 1-9.

[22] Patel VK, Sundaram S, Patel AK and Kalra A. (2018). Characterization of Seven Species of Cyanobacteria for HighQuality Biomass Production. Arabian Journal for Science and Engineering, 43, 109-121.

[23] Verma NM, Mehrotra S, Shukla A and Mishra BN. (2010). Prospective of biodiesel production utilizing microalgae as the cell factories: A comprehensive discussion. African Journal of Biotechnology, 9, 1402-1411.

[24] Řezanka T, Dor I, Prell A and Dembitsky VM. (2003). Fatty acid composition of six freshwater wild cyanobacterial species. Folia Microbiologica, 48, 71-75.

[25] de Swaaf M, Pronk J and Sijtsma L. (2003). Fed-batch cultivation of the docosahexaenoic-acid-producing marine alga Crypthecodinium cohnii on ethanol. Applied Microbiology and Biotechnology, 61, 40-43.

[26] Jiang Y and Chen F. (1999). Effects of salinity on cell growth and docosahexaenoic acid content of the heterotrophic marine microalga Crypthecodinium cohnii. Journal of Industrial Microbiology and Biotechnology, $23,508-513$.

[27] Poisson L and Ergan F. (2001). Docosahexaenoic acid ethyl esters from Isochrysis galbana. Journal of Biotechnology, 91, 75-81.

[28] Liu LN, Chen XL, Zhang YZ and Zhou BC. (2005). Characterization, structure and function of linker polypeptides in phycobilisomes of cyanobacteria and red algae: An overview. Biochimica et Biophysica Acta (BBA) Bioenergetics, 1708, 133-142.

[29] Chen GQ, Jiang Y and Chen F. (2007). Fatty acid and lipid class composition of the eicosapentaenoic acidproducing microalga, Nitzschia laevis. Food Chemistry, 104, 1580-1585.

[30] Sahin D, Tas E and Altindag UH. (2018). Enhancement of docosahexaenoic acid (DHA) production from Schizochytrium sp. S31 using different growth medium conditions. AMB Express, 8, 7.

[31] Ahlgren G, Gustafsson IB and Boberg M. (1992). Fatty Acid Content and Chemical Composition of Freshwater Microalgae. Journal of Phycology, 28, 37-50.

[32] Dawczynski C, Schubert R and Jahreis G. (2007). Amino acids, fatty acids, and dietary fibre in edible seaweed products. Food Chemistry, 103, 891-899.

[33] Calder PC and Yaqoob P. (2009). Omega-3 polyunsaturated fatty acids and human health outcomes. BioFactors, 35, 266-272.

[34] Lopaschuk GD, Ussher JR, Folmes CDL, Jaswal JS and Stanley WC. (2010). Myocardial Fatty Acid Metabolism in Health and Disease. Physiological Reviews, 90, 207-258.

[35] Brostow DP, Odegaard AO, Koh WP, Duval S, Gross MD, Yuan JM and Pereira MA. (2011). Omega-3 fatty acids and incident type 2 diabetes: the Singapore Chinese Health Study. The American Journal of Clinical Nutrition, 94, 520526.

[36] Tiwari A and Kiran T. (2018). Biofuels from Microalgae. In: Nageswara-Rao M and Soneji JR (Eds), Advances in Biofuels and Bioenergy. IntechOpen, 239-249.

\section{How to cite this article}

Şahin OI and Akpınar-Bayizit A. (2020). Determination of the fatty-acid composition of four native microalgae species. GSC Advanced Research and Reviews, 4(1), 01-08. 